\title{
Aliphatic Amines are Viable Pro-drug Moieties in
}

\section{Phosphonoamidate Drugs}

Victoria C. Yan, Cong-Dat Pham, Kenisha Arthur, and Florian L. Muller*

Department of Cancer Systems Imaging, University of Texas MD Anderson Cancer

Center, Houston, TX, 77054, USA

${ }^{*}$ Correspondence should be addressed to Florian L. Muller, Department of Cancer

Systems Imaging, University of Texas MD Anderson Cancer Center, Houston, TX

77054.E-mail:aettius@aol.com
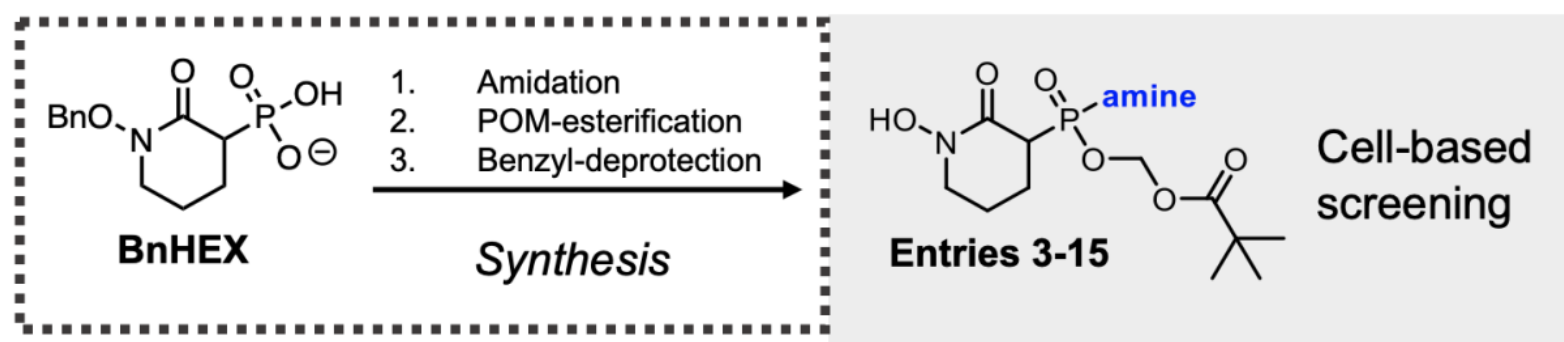
ABSTRACT. Phosphate and phosphonates containing a single P-N bond are frequently used pro-drug motifs to improve cell permeability of these otherwise anionic moieties. Upon entry into the cell, the P-N bond is cleaved by phosphoramidases to release the active agent. Here, we apply a novel mono-amidation strategy to our laboratory's phosphonate-containing glycolysis inhibitor and show that a diverse panel of phosphonoamidates may be rapidly generated for in vitro screening. We show that, in contrast to the canonical L-alanine or benzylamine moieties which have previously been reported as efficacious pro-drug moieties, small aliphatic amines demonstrate greater drug release efficacy for our phosphonate inhibitor. These results expand the scope of possible amine pro-drugs that can be used as second pro-drug leave groups for phosphate or phosphonate-containing drugs.

Phosphoramidates are structurally intriguing chemical moieties with high biological and therapeutic relevance1. Within the realm of drug development, the inclusion of a phosphoramidate moiety has become an increasingly attractive pro-drug strategy for anionic phosphate2,3- or phosphonate4-6- containing drugs. Upon entering the cell, the P$\mathrm{N}$ bond would eventually be cleaved by a class of enzymes known as phosphoramidases 7 ; this includes a family of enzymes known as histidine triad nucleotide-binding proteins (HINT1, HINT2, HINT3)8. From anti-viral drugs such as Tenofovir alafenamideg, Sofosbuvir10, and Remdesivir11 to emerging pro-drugs of conventional chemotherapies such as Gemcitabine (Acelarin12) and 5-Fluorouracil (NUC-3373, NCT0342895813), the phosphoramidate-containing "ProTide" 14 approach to delivering phosphate or phosphonate drugs is a central theme in pro-drug development 1,15 . Common to these 
drugs is the presence of an L-alaninate ester moiety, which is used as a substrate for phosphoramidase cleavage. To the best of our knowledge, apart from the L-alaninate ester, the only other amine that has been reported in the context of phosphoramidate or phosphonoamidate pro-drugs is the benzylamine moiety found in IDX-18416. These observations would suggest that either the L-alaninate or benzylamine moieties would be the most optimal amine substrates for release by phosphoramidases. Fundamental biochemical studies have explored the relationship between altering the amine on phosphoramidate versions of AMP rate of hydrolysis by the phosphoramidases in vitro7,8,17. These studies suggest that amines beyond L-alaninate esters and benzylamine may also be used as second pro-drug groups. Perhaps due to the difficulty in preparing mono-amidated substrates, however, further investigation into the structural limits of optimal amine leave groups in other contexts beyond adenine monophosphate have yet to be reported. Here, we report the efficacy of various structurally diverse amine pro-drug groups in the context of our laboratory's phosphonate inhibitor of the glycolytic enzyme Enolase, termed HEX ${ }_{18}$. We validate the finding that benzylamine and other benzylic amines may be used as second pro-drug groups and also demonstrate that, in the context of HEX, aliphatic amines are often superior delivery moieties. These results may prompt further investigation into using aliphatic amines as pro-drug moieties for phosphate or phosphonate-containing drugs.

We generated a diverse panel of phosphonoamidate pro-drugs of HEX using a novel mono-amidation reaction that we had previously discovered (Yan 2020, manuscript under review). Identifying amines with superior pro-drug properties is a central focus in our 
laboratory's endeavor towards optimizing the delivery of our active Enolase inhibitor, HEX. We previously described an innovative therapeutic strategy, called collateral lethality, wherein cancer cells harboring the homozygous deletion of an essential metabolic gene are selectively sensitized to inhibition of its redundant paralogue5,19. Inhibition of the ENO2 isoform of Enolase in ENO1-deleted cancers results cancerspecific cell death-leaving ENO1-intact or normal cells unperturbed18. To evaluate the relationship between our amine pro-drug structure and efficacy of drug delivery, we first esterified the other free hydroxyl on the phosphonic acid to enable efficient cell permeability (Figure 1a). We chose the pivaloyloxymethyl (POM) group as the first ester group due to its known susceptibility for hydrolysis by ubiquitously expressed carboxylesterases18,20. Importantly, our decision to employ the labile POM ester minimizes the confounding effects of initial pro-drug cleavage and ensures that relationship between amine structure and cell killing can be studied in isolation (Figure 3b). The mono-amidated products generated from our reactions with BnHEX were subject to POM esterification, followed by hydrogenation to liberate the hydroxamate-an absolutely essential moiety for Enolase active site inhibition5,18.

Esterified phosphonoamidates all demonstrated selective, nanomolar activity against ENO1-deleted glioma cells (D423) compared to ENO1-rescued (D423 ENO1) and ENO1wildtype glioma cells (LN319) (Figure 1c). Given the focus on aryl and benzylic amines as pro-drug motifs in the literature 7,21 , we were surprised to find that aliphatic amines proved to be most effective. Cyclopropylmethanamine-protected HEX exhibited 10-fold greater potency compared to benzylamine-protected HEX (IC50, D423 = 22 nM versus 244 
nM, Figure 1c). Direct comparison of benzylamine-protected HEX to its saturated counterpart, cyclohexane methylamine, likewise showed greater potency of the latter (Figure 1c, entry 3 versus 8). Apart from these more striking examples, our data suggest that mono-substitution at the ortho position of the benzylamine ring can enhance cell killing ability and, by extension, hydrolytic activity by phosphoramidases (Figure 1c, entries $4,7,9)$. Substitution at the meta position does not seem to improve the properties of benzylic ring, as observed for the nearly identical $\mathrm{IC}_{50}$ s between benzylamine and 3fluorobenzylamine (Figure 1c, entry 2 versus 5). Collectively, these data show that fluorine substitution on the aromatic ring is well-tolerated, pointing to the feasibility of applying ${ }_{18} \mathrm{~F}$-labeling methods to study pharmacodynamics for any phosphoramidaselabile pro-drug. More generally, we have demonstrated that aliphatic amines-especially those with low molecular weights-are viable pro-drug moieties and can offer superior delivery of phosphonate compounds in vitro (Figure 1, entries 11-15). Taken together, these results point to the viability of using (small) aliphatic amines as pro-drug moieties for phosphates or phosphonates. Compared to L-alaninate esters or benzylamine, small aliphatic amines have lower molecular weights, which could facilitate more efficient cell permeability22. Thus, our findings expand the scope of possible amine pro-drugs that can be used as second pro-drug leave groups. 
A

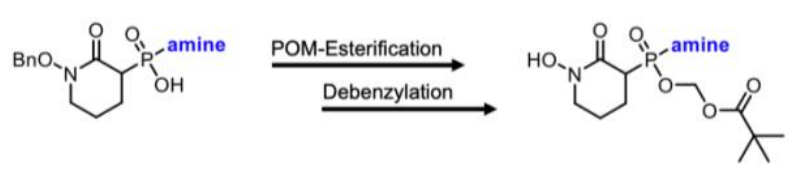

B

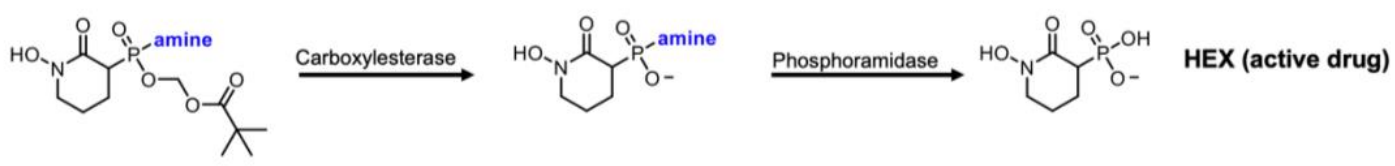

\section{C}

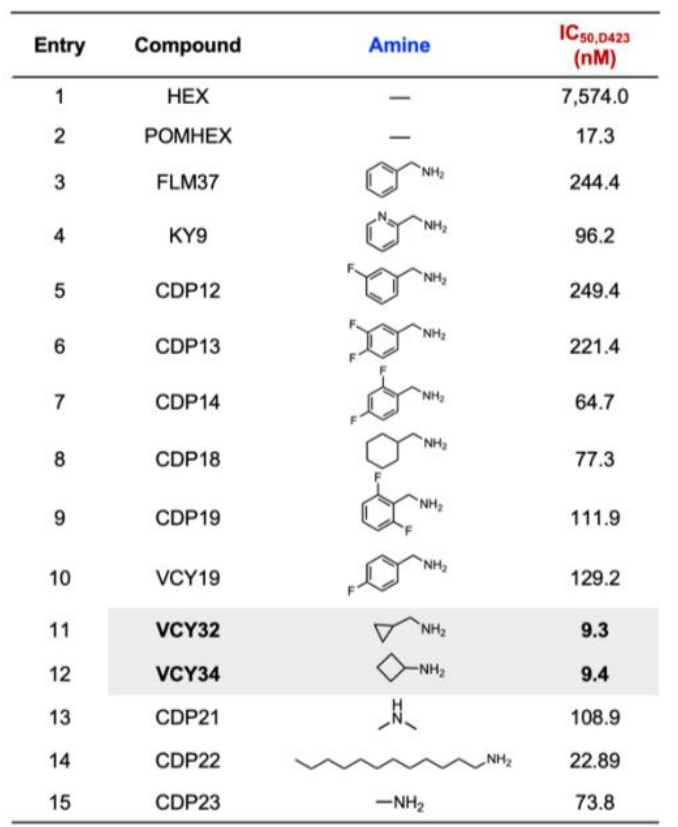
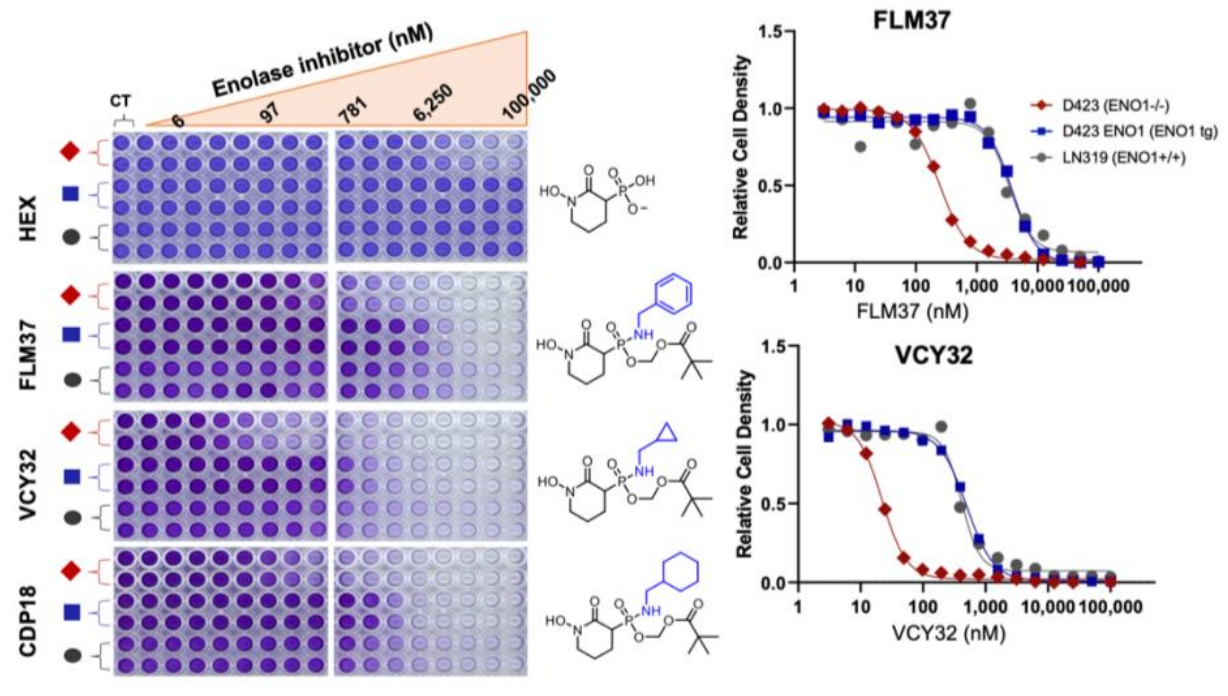

Figure 1. Aliphatic amines offer superior drug delivery in vitro. (a) General workflow for the synthesis and screening amidated pro-drugs. For BnHEX, POM esterification followed by de-benzylation of the hydroxamate yields the final, cell-permeable pro-drug. For specific reaction conditions, see Supplementary Information. (b). Proposed bioactivation mechanism for phosphonoamidate pro-drugs. Phosphoramidases cleave P-N bonds on anionic molecules7 and can thus serve as second pro-drug deprotecting enzymes. (c) The relationship between amine structure and pro-drug efficacy can be evaluated in cell-based screening. (Left) Structures of amine pro-drugs of the Enolase inhibitor, HEX, and corresponding $\mathrm{IC}_{50}$ s against D423 (ENO1-deleted) cells. (Middle) Crystal violet cell proliferation assay evidences greater cell killing against D423 cells by aliphatic amine pro-drugs compared to benzylamine. Cells were incubated with pro-drug inhibitor for 5 days. Then, cells were fixed and stained with crystal violet and quantified spectroscopically. Cell density as measured by crystal violet were plotted as a function of inhibitor. (Right) Comparison of the $\mathrm{IC}_{50}$ values between model aromatic (FLM37) and aliphatic (VCY32) pro-drugs. While both pro-drugs are selective for ENO1-deleted cells, VCY32 exhibits 10 -fold greater potency compared to FLM37 (IC $50=22 \mathrm{nM}$ versus $244 \mathrm{nM})$. 
AUTHOR CONTRIBUTIONS. C.D.P. and V.C.Y performed syntheses and characterized compounds. K.A. performed cell culture experiments. V.C.Y. wrote the manuscript. F.L.M. provided critical comments and oversaw the project.

ACKNOWLEDGEMENTS. This work is supported by the American Cancer Society (RSG-15-145-01-CDD) and the National Comprehensive Cancer Network (YIA170032).

AUTHOR DISCLOSURES. V.C.Y., C.D.P., and F.L.M. are inventors on a patent application describing a novel method for the mono-amidation of phosphates and phosphonates.

\section{REFERENCES}

1. Pradere, U., Garnier-Amblard, E. C., Coats, S. J., Amblard, F. \& Schinazi, R. F. Synthesis of Nucleoside Phosphate and Phosphonate Prodrugs. Chem. Rev. 114, 9154-9218 (2014).

2. McGuigan, C., Cahard, D., Sheeka, H. M., De Clercq, E. \& Balzarini, J. Aryl Phosphoramidate Derivatives of d4T Have Improved Anti-HIV Efficacy in Tissue Culture and May Act by the Generation of a Novel Intracellular Metabolite. J. Med. Chem. 39, 1748-1753 (1996).

3. Bhatia, H. K., Singh, H., Grewal, N. \& Natt, N. K. Sofosbuvir: A novel treatment option for chronic hepatitis C infection. J. Pharmacol. Pharmacother. 5, 278 (2014).

4. Eisenberg, E. J., He, G.-X. \& Lee, W. A. Metabolism of GS-730, A Novel Phenyl 
Monophosphoramidate Intracellular Prodrug of PMPA, In Blood. Nucleosides,

Nucleotides and Nucleic Acids 20, 1091-1098 (2001).

5. Leonard, P. G. et al. SF2312 is a natural phosphonate inhibitor of enolase. Nat.

Chem. Biol. 12, 1053-1058 (2016).

6. Pisaneschi, F. et al. The 3S Enantiomer Drives Enolase Inhibitory Activity in SF2312 and Its Analogues. Molecules 24, 2510 (2019).

7. Krakowiak, A. et al. Biochemical, crystallographic, and mutagenic characterization of hint, the AMP-lysine hydrolase, with novel substrates and inhibitors. J. Biol. Chem. 279, 18711-6 (2004).

8. Bieganowski, P. et al. Adenosine Monophosphoramidase Activity of Hint and Hnt1 Supports Function of Kin28, Ccl1, and Tfb3. J. Biol. Chem. 277, 10852-10860 (2002).

9. Lee, W. A. et al. Selective intracellular activation of a novel prodrug of the human immunodeficiency virus reverse transcriptase inhibitor tenofovir leads to preferential distribution and accumulation in lymphatic tissue. Antimicrob. Agents Chemother. 49, 1898-906 (2005).

10. Sofia, M. J. et al. Discovery of a $\beta$ - $d$-2'-Deoxy-2'- $\alpha$-fluoro-2'- $\beta-C$-methyluridine Nucleotide Prodrug (PSI-7977) for the Treatment of Hepatitis C Virus. J. Med. Chem. 53, 7202-7218 (2010).

11. Siegel, D. et al. Discovery and Synthesis of a Phosphoramidate Prodrug of a Pyrrolo[2,1- $f][$ triazin-4-amino] Adenine $C$-Nucleoside (GS-5734) for the Treatment of Ebola and Emerging Viruses. J. Med. Chem. 60, 1648-1661 (2017).

12. Enrollment in the Independent Investigator-Sponsored Phase III Metastatic 
Pancreatic Study ACELARATE Has Been Suspended Following a Prespecified

Futility Analysis. Globe Newswire (2019).

13. Evans, T. R. J. et al. NuTide:302: A phase lb study to assess the safety, pharmacokinetics and clinical activity of the ProTide NUC-3373 when combined with standard agents used in colorectal cancer. J. Clin. Oncol. 37, TPS719TPS719 (2019).

14. McGuigan, C. et al. Phosphorodiamidates as a Promising New Phosphate Prodrug Motif for Antiviral Drug Discovery: Application to Anti-HCV Agents. J. Med. Chem. 54, 8632-8645 (2011).

15. Alanazi, A. S., James, E. \& Mehellou, Y. The ProTide Prodrug Technology: Where Next? ACS Med. Chem. Lett. 10, 2-5 (2019).

16. Zhou, X.-J. et al. Safety and pharmacokinetics of IDX184, a liver-targeted nucleotide polymerase inhibitor of hepatitis $C$ virus, in healthy subjects.

Antimicrob. Agents Chemother. 55, 76-81 (2011).

17. Chou, T.-F. et al. Phosphoramidate Pronucleotides: A Comparison of the Phosphoramidase Substrate Specificity of Human and Escherichia coli Histidine Triad Nucleotide Binding Proteins. Mol. Pharm. 4, 208-217 (2007).

18. Lin, Y.-H. et al. Eradication of ENO1-deleted Glioblastoma through Collateral Lethality. bioRxiv 331538 (2018). doi:10.1101/331538

19. Muller, F. L. et al. Passenger deletions generate therapeutic vulnerabilities in cancer. Nature 488, 337-343 (2012).

20. Farquhar, D., Khan, S., Srivastva, D. N. \& Saunders, P. P. Synthesis and Antitumor Evaluation of Bis[(pivaloyloxy)methyl] 2,-Deoxy-5-fluorouridine 5- 
Monophosphate (FdUMP): A Strategy To Introduce Nucleotides into Cells. J. Med. Chem. 37, 3902-3909 (1994).

21. SOMMADOSSI, J.-P., GOSSELIN, G., PIERRA, C., PERIGAUD, C. \& PEYROTTES, S. COMPOUNDS AND PHARMACEUTICAL COMPOSITIONS FOR THE TREATMENT OF VIRAL INFECTIONS. (2008).

22. Lipinski, C. A., Lombardo, F., Dominy, B. W. \& Feeney, P. J. Experimental and computational approaches to estimate solubility and permeability in drug discovery and development settings. Adv. Drug Deliv. Rev. 46, 3-26 (2001). 